\title{
Effect on Emotional Well-Being of Hypnotic Recall of the Near-Death Experience
}

\author{
Janice Miner Holden, Ed.D. \\ University of North Texas
}

\begin{abstract}
The prototypical near-death experience (NDE), characterized by positive affect, and its psychologically and spiritually beneficial aftereffects are well documented. However, the aftermath of even a pleasant NDE has sometimes involved psychological difficulties that range from relatively mild adjustment disorders to profoundly distressing and disorienting spiritual emergencies. Consequently, the prospect of recalling an NDE under hypnosis has been fraught with controversy. A recent study (Holden and MacHovec, 1993) suggested that, at least under certain circumstances, hypnotic recall of NDEs could occur without physical or lasting psychological distress. I report here the preliminary finding of overwhelming psychological benefit of such recall, and discuss implications for future research.
\end{abstract}

Although cases of distressing near-death experiences (NDEs) have begun to appear in the professional literature (Atwater, 1992; Greyson and Bush, 1992), the vast majority of reported NDEs have involved predominantly pleasant feelings during, and apparently constructive effects on personality and spirituality after, the experience (Grey, 1985; Ring, 1980, 1984; Sabom, 1982). However, adjustment difficulties, even full-blown spiritual emergencies, sometimes accom-

Janice Miner Holden, Ed.D., is Associate Professor of Counselor Education at the University of North Texas, and maintains a private practice where she specializes in the facilitation of psychospiritual development. The author gratefully acknowledges the assistance of Bruce Greyson, M.D., Research Director of the International Association for Near-Death Studies, and Kenneth Ring, Ph.D., Professor of Psychology at the University of Connecticut, in identifying and contacting potential subjects for the study from among their respective subject pools. Reprint requests should be addressed to Dr. Holden at the Department of Counseling, Development, and Higher Education, University of North Texas, Denton, TX 76203-6857. 
pany even constructive aftereffects of NDEs (Bragdon, 1988; Greyson and Harris, 1989). Therefore, hypnotic recall of a prototypical pleasant NDE might be expected to sustain or enhance positive feelings or aftereffects, respectively.

However, in cases in which psychological difficulties had occurred and remained unresolved, hypnotic recall of the NDE might intensify distress. Even in cases where such difficulties had been resolved or had never occurred, the possibility that hypnotic recall of the NDE might reactivate or precipitate such difficulties could not be ruled out.

A recent study used hypnotic recall of NDEs to address three questions:

1) Can hypnotic recall of the prototypical pleasant NDE occur without harm, that is, without major or lasting physical or psychological distress?

2) What benefit might result from hypnotic recall of the NDE?

3) Can paranormal information be tapped during hypnotic recall of the omniscient phase of the NDE, that phase in which the NDEr experienced a sense of access to any information in the universe?

The first question, of safety, was addressed in a recent publication (Holden and MacHovec, 1993). Under the conditions of this study, hypnotic recall of the NDE occurred without physical detriment to any of the six subjects. In half the cases, subjects experienced no psychological distress associated with NDE hypnotic recall; in the other half, some psychological distress occurred but was quickly resolved.

This article describes findings pertaining to the second question, of potential benefit from hypnotic recall. Because the study has been described in detail elsewhere (Holden and MacHovec, 1993), it will be only briefly summarized here.

\section{Summary of Study}

The primary purpose of the study (Holden and MacHovec, 1993) was to investigate the possible use of "omniscient NDErs," those whose NDEs had contained a phase in which the experiencer perceived him- or herself to have access to any information in the universe, as a source of paranormal information about the genetic basis 
of a hereditary neuromuscular disease. Sixty-two potential subjects were identified from near-death researchers' subject pools and invited to apply for participation in the study. Fifteen respondents were screened for apparent physical and mental health and for presence of the omniscient phase of the NDE. Six subjects, five females and one male ranging in age from 42 to 76 years, participated in the study.

After preliminary contact with the principal investigator, Janice Miner Holden, and the hypnotist, Frank MacHovec, subjects' travel and accommodation expenses were paid for five days at the research site. Subjects were isolated from each other for four days: day 1 for orientation to researchers, facility, and schedule for study, and for psychological screening; day 2 for individual two-hour sessions of general hypnosis induction; and days 3 and 4 each for an individual two-hour session of hypnotic recall of the NDE and, during recall of the omniscient phase, questions pertaining to the primary purpose of the study. On the fifth day subjects and researchers met together to share and process NDE and research experiences.

Most pertinent to the hypnosis procedure is that it did not involve regression. In hypnotic regression, the subject is led from general hypnosis induction through a backwards chronology, stopping at a point of psychological significance, in this case, the subject's NDE. Rather, in this study, subjects were led from general hypnosis induction to a "peaceful place" involving a location and multisensory imagery of the subject's choosing; from there the subject was guided to turn from the peaceful place toward and into the NDE.

After four weeks all subjects were mailed, and all responded to, a follow-up questionnaire. Instructions were as follows:

Please answer the following questions about your sense of emotional well-being regarding hypnotic recall of your NDE. "Negative" effects would include anxiety or other unpleasant emotions that detract from your usual sense of emotional well-being. "Positive" effects would include positive anticipation or other pleasant emotions that enhance your usual sense of emotional well-being.

Feel free to make comments, if you wish, using the back of this sheet.

What was the effect on your sense of emotional well-being regarding hypnotic recall of your NDE at each of the following times?

Fifteen items indicated specific times during the study, arranged chronologically. Likert-type responses ranged from 1 (strongly negative) to 7 (strongly positive). 


\section{Table 1}

Subjects' Ratings of Effect of Hypnotic Recall of NDE on Emotional Well-Being (1 = strongly negative; $7=$ strongly positive)

\begin{tabular}{lcc}
\hline \multicolumn{1}{c}{ Retrospective Time Frame } & Mean & Range \\
\hline anticipating NDE recall during week prior to study & 5.50 & $4-7$ \\
prior to first hypnotic induction on day 1 of hypnosis & 5.67 & $3-7$ \\
after hypnosis session on day 1 of hypnosis & 6.17 & $4-7$ \\
prior to 1st hypnotic induction on day 2 of hypnosis & 5.83 & $4-7$ \\
prior to 1st NDE recall on day 2 of hypnosis & 6.00 & $4-7$ \\
during 1st NDE recall on day 2 of hypnosis & 6.50 & $4-7$ \\
just after 1st NDE recall on day 2 of hypnosis & 5.83 & $3-7$ \\
hours after 1st NDE recall on day 2 of hypnosis & 6.67 & $5-7$ \\
prior to 2nd NDE recall on day 3 of hypnosis & 6.17 & $5-7$ \\
during 2nd NDE recall on day 3 of hypnosis & 6.67 & $6-7$ \\
just after 2nd NDE recall on day 3 of hypnosis & 6.83 & $6-7$ \\
hours after 2nd NDE recall on day 3 of hypnosis & 6.67 & $6-7$ \\
during first day following study & 6.83 & $6-7$ \\
during first week following study & 6.83 & $6-7$ \\
at follow-up 4 weeks after study & 6.67 & $6-7$ \\
\hline
\end{tabular}

\section{Results}

Results of the questionnaire are reported in Table 1 and summarized in Figure 1, where "item number" refers to retrospectively-rated sequential time periods. Respondents perceived hypnotic recall of their NDEs to have an overall positive effect on their sense of emotional well-being. In addition, mean ratings indicated an increasingly positive effect as respondents proceeded through the phases of the study.

\section{Discussion}

The trend for NDErs to rate the effects as increasingly positive as they proceeded through the phases of the study may reflect the re- 
searchers' observation of some anticipatory anxiety that dissipated in the first two days as subjects experienced the hypnosis procedure with greater familiarity and without any or lasting discomfort, and were better able to "relax and enjoy" the process. As previously reported (Holden and MacHovec, 1993), the two ratings of " $3=$ slightly negative" reflected issues that were addressed immediately, resulting in alleviation of distress: one involved anticipatory anxiety about the experience of hypnosis itself, and the other involved the subject feeling self and NDE cruelly denigrated by a minister whom the subject had trusted and respected.

It is noteworthy that another subject's history revealed a difficult adjustment after the NDE, including somewhat extenuated psychotherapy that had ended in a positive outcome some years before the study. Results of psychological screening on the first day of the study indicated that this subject met the criteria for narcissistic personality disorder. The subject experienced a reactivation of some psychologi-

Follow-Up Responses

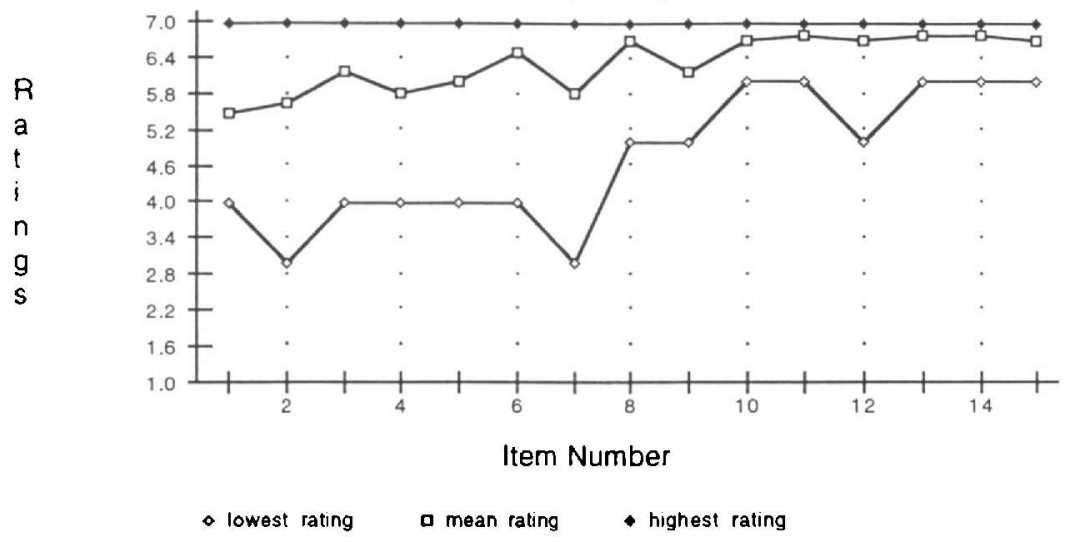

Figure 1. Subjects' ratings of effect of hypnotic recall of NDE on emotional well-being ( $1=$ strongly negative; $7=$ strongly positive). 
cally distressing material after her first hypnotic NDE recall: at the time of the NDE, the subject had wanted the experience to continue, and in the aftermath of the NDE she felt guilty about having been willing to abandon her family. The researchers helped this subject to work through this material rather quickly, and observed an apparently complete alleviation of psychological distress. Interestingly, the subject's follow-up ratings consisted entirely of 6's and 7's, making no reference to the transient detraction from her sense of emotional well-being.

In summary, the NDErs in this study, most of whom evidenced no adjustment difficulties in the aftermath of their prototypical pleasant NDEs and one of whom evidenced both adjustment difficulties that had been resolved and an ongoing personality disorder, reported hypnotic recall of their NDEs to have overall a quite positive effect on their sense of emotional well-being. In three cases in which the researchers observed psychological distress surrounding hypnosis or NDE recall, the distress was addressed and worked through immediately, had no lasting effect on sense of emotional well-being, and was reported in follow-up by only two of the three subjects.

For a number of reasons, the results reported herein must be considered preliminary. The sample size was small. Only subjects who expressed primarily positive anticipation of NDE recall were included. Subjects were carefully screened for physical and psychological health. A specific and standardized hypnotic procedure was used. Although subjects were asked to consider only the isolated factor of hypnotic recall of their NDE in responding to the follow-up questionnaire, they may have had difficulty isolating this variable from other variables.

For example, repeated hypnosis alone might account for an enhanced sense of emotional well-being; conversely, repeated, purposeful recall of the prototypical pleasant NDE without hypnosis may be responsible for the effect; and the positive set of being selected to participate in an all-expenses-paid study may very well have shed a halo effect on subjects' overall sense of emotional well-being before, during, and after the study.

On the other hand, some anecdotal material supports the hypothesis that hypnotic recall of a pleasant NDE may be psychologically beneficial. One subject reported that after her NDE, when she felt overwhelmed by the stress of her prolonged, very difficult life circumstances, she would occasionally isolate herself and, using relaxa- 
tion and focusing techniques not unlike hypnosis, would draw on her memory of her NDE. On those occasions, she found the memory or reexperience of her NDE to be a source of sustenance and renewal, in essence, extremely therapeutic.

In addition, five of six subjects in this preliminary study specifically asked to be given an audiotape of their personalized NDE-recall hypnosis induction. It appears that NDErs who did not spontaneously discover but were introduced to the practice showed a high degree of interest in continuing it, reportedly because they found it subjectively pleasant and/or beneficial.

Their reports of pleasantness/benefit might be understood in light of recent findings that dogs who were conscious at the moment of cardiac arrest showed a significant increase in $\beta$-endorphin, one of the body's endogenous opioids (Sotelo, Perez, Guevara, and Fernandez, 1995). Hypnotic recall of the NDE may constitute a stimulus for the conditioned response of $\beta$-endorphin release, which would hypothetically result in the subjective experience of pleasure.

This supportive material is interesting to consider in light of other research findings that NDErs do not report greater satisfaction with life (Greyson, 1994; Olson and Dulaney, 1993). It may be, for example, that NDErs do not "naturally" draw on NDE recall, but if they did, they might experience greater life satisfaction along with greater emotional well-being. Another possibility requires a closer look at items on the Satisfaction With Life Scale (Diener, Emmons, Larson, and Griffin, 1985). For example, an NDEr may not report any greater frequency of having "gotten the important things I want in life" (Diener, Emmons, Larson, and Griffin, 1985, p. 72), but may feel more emotional well-being in the face of that situation. Further research may tell.

The question of benefit of hypnotic recall of pleasant NDEs could be further researched also by using a larger sample of NDErs, not limited to omniscient NDErs, and comparing conditions that would isolate the necessary elements for positive effects. A related question is whether hypnotic recall of pleasant near-death-like experiences (Holden and Guest, 1990) also might yield positive effects on emotional well-being. Now that the safety of hypnotic recall, at least under the conditions described in this study, has been established, and the benefit of such recall to experiencers seems likely, further research, following the guidelines described by Holden and MacHovec (1993), may be undertaken with greater confidence. 


\section{References}

Atwater, P. M. H. (1992). Is there a hell? Surprising observations about the near-death experience [Guest editorial]. Journal of Near-Death Studies, 10, 149-160.

Bragdon, E. (1988). A sourcebook for helping people in spiritual emergency. Los Altos, CA: Lightening Up Press.

Diener, F., Emmons, R. A., Larson, R. J., and Griffin, S. (1985). The Satisfaction With Life Scale. Journal of Personality Assessment, 49, 71-75.

Grey, M. (1985). Return from death: An exploration of the near-death experience. London, England: Arkana.

Greyson, B. (1994). Near-death experiences and satisfaction with life. Journal of NearDeath Studies, 13, 103-108.

Greyson, B., and Bush, N. E. (1992). Distressing near-death experiences. Psychiatry, 55, 95-110.

Greyson, B., and Harris, B. (1989). Counseling the near-death experiencer. In S. Grof and C. Grof (Eds.), Spiritual emergency: When personal transformation becomes a crisis (pp. 199-210). Los Angeles, CA: Tarcher.

Holden, J. M., and Guest, C. (1990). Life review in a non-near-death episode: A comparison with near-death experiences. Journal of Transpersonal Psychology, 22, 1-16.

Holden, J. M., and MacHovec, F. (1993). Risk management in hypnotic recall of neardeath experiences. American Journal of Clinical Hypnosis, 36, 38-46.

Olson, M., and Dulaney, P. (1993). Life satisfaction, life review, and near-death experiences in the elderly. Journal of Holistic Nursing, 11, 368-382.

Ring, K. (1980). Life at death: A scientific investigation of the near-death experience. New York, NY: Coward, McCann and Geoghegan.

Ring, K. (1984). Heading toward omega: In search of the meaning of the near-death experience. New York, NY: Morrow.

Sabom, M. B. (1982). Recollections of death: A medical investigation. New York, NY: Harper and Row.

Sotelo, J., Perez, R., Guevara, P., and Fernandez, A. (1995). Changes in brain, plasma and cerebrospinal fluid contents of $\beta$-endorphin in dogs at the moment of death. Neurological Research, 17, 223-225. 